\title{
Edna Foa: Das Besondere bei PTSD ist, dass die Betroffenen Angst haben, an das traumatische Ereignis zu denken
}

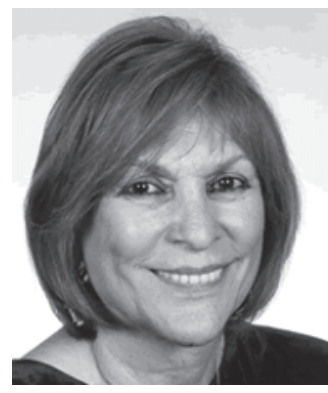

Keine Frage, Edna Foa gehört zu den Großen der Verhaltenstherapie. Sie ist Professorin für Klinische Psychologie und Psychiatrie an der Universität von Pennsylvania, Direktorin des "Center for the Treatment and Study of Anxiety" und eine international anerkannte Autorität auf dem Gebiet der Angststörungen. Sie verbindet in vorbildlicher Weise psychotherapeutische Praxis mit exzellenter wissenschaftlicher Forschung. In einer Zeit, in der Ängste und Angststörungen auf dem Vormarsch sind, ist ihr Werk von besonderer gesellschaftlicher Bedeutung. Ihre Forschung hat das heutige Verständnis und die Behandlung der Zwangsstörungen und der posttraumatischen Belastungsstörungen entscheidend geprägt. Die Bandbreite ihrer Arbeiten reicht von experimentellen Grundlagenstudien über die Entwicklung ätiologischer Modelle bis zur Überprüfung psychologischer Behandlungsansätze bei Zwangsstörungen und posttraumatischen Belastungsstörungen. Insbesondere ihre Behandlungsprogramme gelten als Goldstandard und werden weltweit eingesetzt. Dass sie neben ihrer wissenschaftlichen und therapeutischen Arbeit immer Zeit für ihre drei Töchter und für intensiven Kulturgenuss gefunden hat, macht Edna Foa zudem für viele jüngere Kolleginnen und Kollegen zu einem begeisternden Vorbild. Das Interview führte Prof. Dr. Jürgen Margraf, Basel.

$\mathrm{Zu}$ allererst würde ich Sie gerne fragen, wie es kam, dass Sie von der Sozialpsychologie zur klinischen Psychologie wechselten?

Edna Foa: Eigentlich habe ich gar nicht von der Sozialpsychologie zur klinischen Psychologie gewechselt. Ich habe meinen Bachelor 1962 an der Bar-Ilan-Universität in Israel in klinischer Psychologie gemacht. Damals konnte man damit in Israel auch wirklich als klinischer Psychologe arbeiten, und das tat ich. Ich machte viele diagnostische Interviews und viel Psychotherapie, vor allem psychodynamische Psychotherapie. Die kognitive Verhaltenstherapie habe ich eigentlich erst viel später kennen gelernt. Ich habe ungefähr 3 Jahre lang als klinische Psychologin gearbeitet. Dann sind wir in die USA gezogen, und ich beschloss, einen $\mathrm{PhD}$ zu machen, um auch wissenschaftlich arbeiten zu können.

Wann haben Sie «Societal Structures of the Mind» mit Ihrem Ex-Ehemann veröffentlicht?

Edna Foa: 1974. Eigentlich machte ich meinen Master in Psychopathologie, nicht in Sozialpsychologie. In meiner Master-Arbeit verglich ich schizophrene mit depressiven Leuten, also ein sehr klinisches Thema. Dann zog ich um, weil mein Ex-Ehemann eine Forschungsprofessur an der Universität von Missouri erhielt. Dort setzte ich meine akademische Ausbildung fort. Damals fand ich das Lehrprogramm in klinischer Psychologie nicht besonders interessant, die Sozialpsychologie schien mir viel interessanter. Also verschob ich mein Interesse Richtung Sozialpsychologie, aber auch wenn meine Abschlussarbeit in Persönlichkeits- und Sozialpsychologie war, hatte sie viel mit klinischer Psychologie zu tun.

Also gab es eigentlich mehr Kontinuität, als ich dachte. Dann haben Sie sich in der klinischen Psychologie und Psychotherapie sehr engagiert und arbeiteten zu Zwangsstörungen. Aber ich möchte Sie zunächst nach Ihrer Arbeit zur posttraumatischen Belastungsstörung fragen. Wie kamen Sie dazu?

Edna Foa: Es war im Gegensatz zu meiner Arbeit ziemlich interessant. Mein Interesse konzentrierte sich aber weniger auf Zwangsstörungen oder PTSD als auf Angststörungen. Als wir direkt nach meinem PhD nach Philadelphia zogen, entdeckte ich, dass Wolpe in Philadelphia war. Obwohl ich mich damals gar nicht so für Verhaltenstherapie interessiert hatte, hatte ich in Illinois viele Veranstaltungen in Verhaltensthera-

\begin{tabular}{|c|c|c|}
\hline KARGER & (C) 2005 S. Karger GmbH, Freiburg & $\begin{array}{l}\text { Prof. Dr. rer. soc. Jürgen Margraf } \\
\text { Institut für Psychologie }\end{array}$ \\
\hline $\begin{array}{l}\text { Fax +49 } 7614520714 \\
\text { E-mail Information@Karger.de } \\
\text { www.karger.com }\end{array}$ & $\begin{array}{l}\text { Accessible online at: } \\
\text { www.karger.com/ver }\end{array}$ & $\begin{array}{l}\text { Universität Basel } \\
\text { Missionsstr. 60-62, } 4055 \text { Basel, Schweiz } \\
\text { Tel. +41 61 26706-60, Fax -48 } \\
\text { E-mail juergen.margraf@unibas.ch }\end{array}$ \\
\hline
\end{tabular}


pie belegt. Illinois war ein Mekka der Verhaltenstherapie. Ich dachte, wenn Wolpe so genial war, dass er der Vater der Verhaltenstherapie wurde, muss er ein sehr interessanter Mensch sein. Also beschloss ich, einen Antrag zu stellen für die Finanzierung einer Post-Doc-Stelle bei Wolpe. So kam ich zur Verhaltenstherapie und zu Verhaltensstörungen. Meine Arbeit zu Zwangsstörungen war wirklich Zufall und kam dadurch, dass meine erste Patientin bei Wolpe unter einer Zwangsstörung litt. Es war die schwierigste Patientin, die ich je hatte.

\section{Und systematische Desensibilisierung funktionierte nicht?}

Edna Foa: Nein, das war interessant, denn diese Patientin war vorher bei Wolpe, bevor er nach Philadelphia kam. Er hatte systematische Desensibilisierung mit ihr versucht, aber es half nicht. Sie folgte ihm nach Philadelphia und war dort die erste Patientin, um die ich mich kümmerte. Wir probierten systematische Desensibilisierung. Sie fühlte sich stark angezogen von allem, was mit dem Tod zusammenhing, und hatte keine Angst vor dem Tod; aber vor dem Vergiftetwerden. Außerdem hatte sie Angst vor allem, was mit dem Tod zu tun hat, wie vor Friedhöfen oder vor Blumengeschäften, weil man dort Blumen für Begräbnisse kaufen kann. Wenn sie auf der Straße einen Leichenwagen sah, fühlte sie sich beschmutzt. - Ich sollte also systematische Desensibilisierung anwenden, indem ich in einem Friedhof einen Stein aufheben sollte, ihn 20 Fuß vor sie hinlegen und warten sollte, bis sie sich entspannte fühlte, um anschließend den Stein einen halben Fuß näher zu legen. Das ging drei Monate so. Ich ging jeden Tag zu ihr, und diese Frau war so gestört, dass sie nackt im Bett lag.

\section{Um nicht beschmutzt oder vergiftet zu werden?}

Edna Foa: Genau. Sie hatte ein Leintuch über sich und erlaubte niemandem, sie zu berühren. Sie konnte nicht einmal Essen berühren, so dass ihr Mann sie füttern musste, damit sie nichts anfassen musste. Das einzige, was sie sich zu berühren erlaubte, war die Fernbedienung für den Fernseher.

\section{Eine sehr grundlegendes Bedürfnis.}

Edna Foa: Ja. Als sie nach drei Monaten keinerlei Fortschritt gemacht hatte, kam Wolpe als mein Supervisor mit und erklärte ihr, dass es zwei neue Behandlungen gebe. Eine davon wäre Flooding, wobei man im Gegensatz zur bisherigen, schrittweisen Behandlung alles viel schneller machen würde, so dass sie Sachen machen müsste wie zum Friedhof gehen und einen kleinen Stein holen etc.; die andere Behandlung wäre eine Hirnoperation. Er sagte ihr, Flooding sei sehr gefährlich. Auf die Cingulotomie ging er nicht näher ein. Also entschied sich die Patientin für die Operation. Nachdem Wolpe gegangen war, fragte ich sie, ob sie nicht Angst hätte, dass man in ihrem Gehirn herumwühlen würde. Sie meinte, sie habe große Angst, aber weniger Angst als davor, zum Friedhof zu gehen. Sie unterzog sich zwei Cingulotomien, die ihr überhaupt nicht halfen, und kam ein Jahr später zurück und machte, was wir damals Flooding nannten und heute Konfrontationstherapie mit Reaktionsverhinderung, und das hat ihr sehr geholfen. - Aber was PTSD angeht, ich wusste wirklich nicht viel über PTSD, niemand wusste viel darüber. Mein Interesse begann in den 80er Jahren. Ich kam aus theoretischen Überlegungen dazu, weil ich mich fragte, wie Menschen sehr beängstigende Ereignisse bewältigen können. Ich wollte also Angst verstehen.

Gefährliche oder erschreckende Ereignisse im Vergleich zu einem Zwangspatienten, der sich vor Verschmutzung durch ein Blumengeschäft fürchtet?

Edna Foa: Ich bin nicht sicher, ob sich die Stärke der Angst unterscheidet, aber ich dachte, traumatische Erfahrungen wären ein gutes Beispiel, um zu verstehen, wie Menschen konditioniert werden, wie sie das überwinden und wie sie nicht konditioniert werden. Eigentlich galt mein Interesse also der emotionalen Verarbeitung, wie Menschen verarbeiten. Wenn ich die Prozesse und Mechanismen bei der Bewältigung eines sehr beängstigenden Ereignisses verstehe, und eine traumatische Erfahrung ist ein sehr beängstigendes Ereignis, wenn ich verstehe, wie Menschen ein Trauma erleben und was dabei mit ihnen passiert, dann kann ich auch untersuchen, was mit ihnen geschieht, wer sich von einem Trauma erholt und wer nicht; dann kann man den Mechanismus, der einer Angststörung zugrunde liegt, verstehen.

Das scheint mir kennzeichnend für Ihre Arbeit zu sein. Dieses sehr grundlegende Interesse daran, zu verstehen, was passiert, nicht nur daran, eine emotionale Heilung zu erreichen.

Edna Foa: Zumindest am Anfang meiner Karriere wollte ich vor allem die Mechanismen pathologischer Angst verstehen, erst später dann die Mechanismen einer erfolgreichen Behandlung. Der interessanteste Teil meiner Arbeit war, wer auf die Therapie anspricht und wer nicht. Ich war am Anfang weniger am Resultat der Behandlung interessiert.

Man könnte also sagen, dass Sie sehr erfolgreich waren mit Zwangsstörungen und dass vor allem Ihr Wunsch, pathologische Angst besser zu verstehen, Sie bewog, sich auf eine anderes Krankheitsgebiet zu spezialisieren, also ein eher theoretisches als therapeutisches Interesse. Wenn wir jetzt aber auf Therapie zu sprechen kommen, was sollten Therapeuten bei einem PTSD-Patienten Ihrer Meinung nach zuerst versuchen?

Edna Foa: Denken Sie daran, dass die PTSD 1980 erfunden wurde. Denken Sie daran, dass viele damals gar nicht wussten, dass es posttraumatische Reaktionen gibt, auch wenn das Interesse und der Fokus auf posttraumatischen Reaktionen und deren Behandlung lag. Deren Therapie begann mit der Einführung der Kategorie Angststörung in das DSM-III 1980. Das war vermutlich ein Resultat der politischen Verhältnisse zur Zeit des Vietnamkriegs. Kriegsveteranen hatten diese Symptome, die nicht weggingen. Dann gab es einen gewissen politischen 
Druck, die Symptome im DSM-III zu operationalisieren, und plötzlich interessierten sich alle dafür. Mein Interesse an pathologischer Angst und Traumata rückte in den Vordergrund, plötzlich gab es da ein eigenes Krankheitsbild. Vorher dachte ich, eine traumatische Erfahrung wäre einfach ein ...

\section{... ein Risikofaktor?}

Edna Foa: Eine Form der einfachen Phobie. Was hätte es sonst sein sollen? - Sie sind ein normaler Mensch, haben keine bestimmten Ängste, gehen in den Krieg, fliegen, verunglücken mit dem Flugzeug, und entwickeln danach intensive Furcht und Vermeidungsverhalten, also genau das, was eine einfache Phobie ausmacht. Aber dann haben wir herausgefunden, dass das keine Phobie ist, sondern posttraumatischer Stress. Und jetzt müssen wir verstehen, wie man diesen behandelt und warum sich manche davon erholen und manche nicht. Ich dachte von Anfang an, dass der Mechanismus, der der Erholung zugrunde liegt, vermutlich ähnlich oder gleich ist, wie jener der Behandlung. Die meisten Menschen erholen sich von einem traumatischen Ereignis wieder. Dann entwickelte ich die Theorie, dass man sich im Allgemeinen von einem Trauma erholt, wenn man möglichst normal weiterlebt und sich bewusst wird, dass traumatische Ereignisse wirklich ziemlich selten sind, zumindest für jeden einzelnen. Also überlegte ich mir, dass die Leute, die sich nicht erholen, diejenigen sind, die nicht normal weiterleben und an unrealistischen Vorstellungen über die Welt festhalten,

\section{... unmittelbare Gefahr ...}

Edna Foa: ... dass die Welt sehr gefährlich ist, dass jeden Moment etwas passieren kann. Außerdem vermutete ich, dass diese Menschen meinen, damit nicht fertig werden zu können, dass das Geschehene so überwältigend war, dass sie nie wieder so zurechtkommen wie früher und nicht mehr derselbe sind. Also schloss ich: Wenn Vermeidung die Symptome aufrechterhält, dann müssen wir Konfrontation machen. Damit hatte ich ja auch Erfahrung. Unter Wolpe hatten wir eine Angstambulanz mit vielen traumatisierten Patienten. Wir wussten damals nur nicht, dass sie PTSD hatten. Es waren Phobiker, mit denen wir Konfrontationstherapie machten. Wir konfrontierten sie mit ihren traumatischen Erinnerungen und waren sehr erfolgreich. Als daher PTSD als eigene Diagnose auftauchte, schien es mir nur natürlich, die Arbeit, die ich bei Zwangs- und Angstpatienten gemacht hatte, auch mit PTSD-Patienten zu machen. PTSD ist im Allgemeinen eine Angststörung. Daher müssten die Behandlungen, die bei Angststörungen helfen, auch bei PTSD greifen. Das Neue, das ich gemacht habe, war mein Versuch herauszufinden, was das Besondere bei PTSD ist, und zu zeigen, wie die Konfrontationstherapie in diesem Fall durchgeführt werden sollte.

Ein wichtiger Punkt dabei ist das Gedächtnis. Die Therapie muss den Patienten helfen, das Trauma zu verarbeiten und es ins autobiographische Gedächtnis zu integrieren und sich nicht mehr so zu verhalten als wäre es immer noch Teil des momentanen Lebens. Was meinen Sie genau mit Ins-Gedächtnis-Integrieren?

Edna Foa: Patienten mit quälender Angst fürchten sich davor, dass ihnen bestimmte schlimme Sachen geschehen. Ihre Furcht ist unrealistisch, denn sie überschätzt die reale Gefahr vollkommen. Wer eine soziale Phobie hat, meint, es sei schrecklich, wenn die Leute ihn nicht für superintelligent halten und er nicht den allerbesten Vortrag hält. Wenn Sie solche negativen Gedanken haben oder ständig Situationen auf diese Weite bewerten, kann das ihr Leben sehr beeinträchtigen. Also überlegte ich mir, wovor PTSD-Patienten Angst haben. Ich achtete darauf, wie sie sprechen, und erkannte, dass sie Angst haben, es könnte etwas Schreckliches passieren und dass sie sich nicht mehr unter Kontrolle hätten, wenn sie sich erlauben, an das traumatische Ereignis zu denken. Daher versuchen sie, das traumatische Geschehen aus ihren Gedanken zu verbannen. Aber es verfolgt sie in Form von Flashbacks oder Intrusionen trotzdem. Sie stellen sich ihren Erinnerungen nicht lange genug, um sie in einen sinnvollen Zusammenhang bringen zu können und das Erlebte richtig einzuordnen, zu reorganisieren, aus der gegenwärtigen Perspektive als Erinnerung zu betrachten und es dann beiseite zu legen, um es im Langzeitgedächtnis zu speichern, so wie man es mit anderen Erinnerungen macht. Einige Gedächtnistheorien sagen, dass traumatische Erinnerungen die ganze Zeit im Arbeitsgedächtnis bleiben. Dort sind sie einerseits sehr leicht abzurufen, werden aber andererseits ignoriert. Daher ist ein zentraler Aspekt der Konfrontation, die Erinnerung zuzulassen und sich ihr so lange zu stellen, dass sie neu erzählt werden kann.

Für diese Theorie spricht ja auch, dass traumatische Erinnerungen meistens fragmentiert sind.

Edna Foa: Zumindest die Informationen, die unter massiver Angst enkodiert werden, sind fragmentiert. Bittet man die Patienten, ihre Geschichte zu erzählen, stellt man auch fest, dass sie ganz zerhackt erzählen, nicht so, wie man beispielsweise erzählt, was einem gestern passiert ist, als man mit einem Freund Essen war - dann erzählt man eine kohärente Geschichte, die zeitlich und räumlich einer bestimmten Abfolge gehorcht. Patienten mit traumatischen Erinnerungen erzählen keine schlüssige Geschichte, es fehlt der zeitliche und räumliche Faden. Wir haben einige Studien durchgeführt, um zu prüfen, ob die Organisation der Erzählungen besser wird, je öfter sie erzählt werden. Und tatsächlich, der Grad der Organisiertheit der Erzählung hing mit dem Behandlungsergebnis zusammen, wie wir vermutet hatten.

Ich möchte noch einmal auf das Vermeiden zurückkommen. Gibt es Ihrer Meinung nach spezielle Unterschiede bei der Vermeidung bei den verschiedenen Angststörungen? Früher sagte man, dass Phobiker vermeiden, andere Angstpatienten nicht. Diese Tage wissen wir, dass auch PTSD-Patienten und Zwangs- 
patienten vermeiden; sogar Patienten mit generalisierterAngststörung zeigen kognitive Vermeidung. Ist es sinnvoll, zwischen diesen unterschiedlichen Angststörungen zu unterscheiden?

Edna Foa: Man muss unterscheiden zwischen kognitiver Vermeidung, die PTSD-Patienten zeigen, um unangenehme Erinnerungen $\mathrm{zu}$ vermeiden oder zu ignorieren, indem sie sich ablenken und an andere Sachen denken. Daneben gibt es die Vermeidung von Situationen, die einen an das Trauma erinnern könnten oder die dem Trauma so ähnlich scheinen, dass man fürchtet, es könnte wieder etwas Schreckliches geschehen. Das sind zwei unterschiedliche Dinge: Sie können eine Situation vermeiden, nicht nur, weil Sie denken, dass etwas Schreckliches passieren könnte, sondern auch, weil Sie nicht an das Trauma erinnert werden wollen. Der Mechanismus dabei ist ähnlich wie bei der kognitiven Vermeidung.

Das Vermeiden von Situationen, weil wieder etwas Schreckliches passieren könnte, ähnelt dem Vermeidungsverhalten einfacher Phobien. Wer eine Hundephobie hat, geht nicht auf Plätze mit vielen Hunden, weil ihn ein Hund beißen könnte. Leute mit PTSD haben Angst, abends um acht aus dem Haus zu gehen, weil sie wieder vergewaltigt werden könnten. Bei der In-vivo-Exposition konfrontieren wir die Patienten mit realen Situationen, nicht mit Erinnerungen. Es ist aber sehr wichtig, genau zu verstehen, wovor jemand Angst hat. PTSDPatienten haben oft Angst vor der Erinnerung. Dann muss die Konfrontation anders aussehen, als wenn die Angst ist, dass ich wieder gepackt werden könnte. Vermeidung kann aber noch viel unterschiedlicher sein. Denken Sie an Zwangspatienten. Diese vermeiden Situationen, die sie «beschmutzen» könnten oder in denen ihnen etwas Schlechtes zustoßen könnte oder die sie nicht ertragen könnten. Aber da es sehr schwierig ist, all diese Situation zu vermeiden, entwickeln Zwangspatienten ein rituelles Verhalten.

\section{Oder magisches Verhalten ...}

Edna Foa: ... was rituellem Verhalten entspricht, den Zwangshandlungen. Experimentelle Psychologen bezeichnen Zwangshandlungen als aktive Vermeidung im Gegensatz zu passiver Vermeidung, das man eher Fluchtverhalten nennen würde. Bei Zwangspatienten müssen Sie darauf achten, die Patienten am Ausführen ihres aktiven Vermeidungsverhaltens zu hindern, nicht nur an der passiven Vermeidung. Bei PTSDPatienten beinhaltet Exposition meistens die Konfrontation mit Situationen, die sie passiv vermeiden. Bei Patienten mit sozialer Phobie ist das Besondere, dass sich soziale Situationen nicht vollständig vermeiden lassen. Diese Patienten zeigen häufig ein gewisses Sicherheitsverhalten, auch das ist eine Art von Vermeidung.

Dank Ihrer Arbeit haben wir heute auch für Störungen wie Zwänge und PTSD wirkungsvolle Behandlungsmethoden. Sie haben weiterentwickelt, was bei Phobien oder anderen Angststörungen schon vorher gemacht wurde. Nun zu einem ganz anderen Thema: Neben Ihrer ganzen wissenschaftlichen Kar- riere, haben Sie auch drei Kinder aufgezogen. Gab es da je einen Konflikt? War das schwierig? Hatten Sie ein Vorbild?

Edna Foa: Nein, eigentlich hatte ich kein Vorbild dafür. Aber ich wuchs in Israel in einem Umfeld und zu einer Zeit auf, zu der eine Art westlicher Glaube an die Möglichkeiten der Sozialisation vorherrschte und die Überzeugung bestand, dass Männer und Frauen die gleichen Sachen machen können. Daher gab es keine so strenge Unterscheidung zwischen dem, was Frauen erlaubt war, und dem, was Männern erlaubt war. Zumindest nicht für mich. Ich glaube, auch meine Eltern haben mir vermittelt, dass Frauen sich nicht anders verhalten sollten als Männer.

Also war es für Sie normal, sowohl Karriere zu machen als auch Kinder zu haben?

Edna Foa: Ja. Ich bekam mein erstes Kind, zwei Monate bevor ich meinen Bachelor machte. Meine zweite Tochter kam in den Semesterferien, als ich an meiner Promotion saß, und ich nahm einen Monat frei. Danach ging ich zurück zur Uni. Und dann kam mein letztes Kind, als ich eigentlich einen Vollzeitjob hatte. Da nahm ich 10 Tage frei.

10 Tage ...

Edna Foa: Danach ging ich für eine kurze Zeit für 2, 3 Stunden zurück, und innerhalb von zwei Monaten war ich Vollzeit zurück. Ich hatte immer Haushaltshilfen, die auch bei uns wohnten. Insofern sah ich mich wirklich die gleichen Sachen machen wie Männer: Sie haben zwar ein Baby, aber deswegen müssen Sie nicht aufhören zu arbeiten.

Sie gehören vermutlich zu den ganz wenigen Frauen, die das gemacht haben. Dabei gibt es viele Männer, die Kinder haben, aber dann kümmern sich meistens ihre Frauen um die Kinder.

Edna Foa: Ich denke, Sie haben vollkommen Recht. Ich weiß niemanden - nein, ich hatte Freunde, die Kinder hatten, aber sie opferten einen Teil ihrer Karriere. Sie stellten sicher, dass sie um ein Uhr wieder zu Hause waren, damit die Kinder das richtige Essen bekämen. Ich überließ dies Haushaltshilfen. Ich war um fünf zu Hause und verbrachte den Abend und die Ferien mit meinen Kindern.

Diese Haushälterinnen, waren das Au-Pair-Mädchen oder Frauen, die Sie anstellten, oder wie sah das aus?

Edna Foa: Ich hatte das Glück, 10 Jahre lang die gleiche Haushälterin zu haben. Ich brachte sie aus Israel mit. Ich glaube, es war auch hilfreich, dass mein damaliger Mann an einem anderen Punkt seiner Karriere stand, er war schon weiter. Daher war es für ihn einfacher, zu Hause zu bleiben. Er arbeitete zwar ganztags an der Universität, aber er blieb häufiger zu Hause als ich. Aber ich habe nicht viel darüber nachgedacht. 
Also schien Ihnen das Ganze eine natürliche Lösung zu sein, obwohl die meisten es anders machten?

Edna Foa: Ja. Ich bin immer wieder erstaunt, wenn junge Frauen sagen, dass ich ein Vorbild sei, und wissen wollen, wie ich das gemacht habe. Dann sage ich, weil ich es mir nicht bewusst gemacht habe, nicht vor Augen gehalten habe, dass ich eine Frau bin. Ich dachte, wenn Männer das machen, wo ist der Unterschied? Auf diese Weise fühlte ich mich nicht schuldig.

Wie war es mit dem männlichen Umfeld? Es gibt ja immer Netzwerke, möglicherweise nicht sehr offen, aber es gibt Kontakte. War es für Sie schwierig, ein Teil des «Old Boy Netzwerkes» zu sein?

Edna Foa: Ich glaube, ja. Ich glaube, dass ich mir auch dessen nicht allzu bewusst war. Gerade weil mir nicht so bewusst war, dass ich Sachen anders mache als andere Frauen. Aber hin und wieder gab es Situationen, die mich daran erinnerten, dass ich manchmal, in bestimmten Fällen nicht dazugehörte. Im Nachhinein denke ich, dass es vermutlich für mich schwieriger war, und vielleicht musste ich auch härter arbeiten. Aber ich war nicht sehr besorgt. Es kümmerte mich auch nicht, dass ich Ausländerin, Immigrantin war.

Könnte es sein, dass die Tatsache, dass Sie sich so, in Anführungszeichen «natürlich»verhalten haben, dazu führte, dass Sie nicht bitter wurden, weil Sie keine Gedanken daran verschwendeten?

Edna Foa: Genau. Ich glaube, ich habe wirklich erst viel später, vielleicht vor 10 Jahren, angefangen darüber nachzudenken, dass es vermutlich Situationen gab, in denen ich einflussreicher hätte sein können, besonders im politischen Bereich, bei berufspolitischen Sachen. Wahrscheinlich war es für mich manchmal schwieriger, berufspolitisch mitzureden, aber ich habe mir keine großen Sorgen darum gemacht.

Ich habe es aber auch so erlebt, dass Sie an Politik weniger interessiert waren.

Edna Foa: Vielleicht war ich weniger interessiert, vielleicht war ich aber auch nur weniger interessiert, weil es schwieriger war, es war weniger möglich. Ich neige dazu, zuversichtlich zu sein in Bezug auf meine Aussichten und konzentriere mich auf das, was ich am besten kann. Ich fand immer, dass ich im Kombinieren von theoretischen und therapeutischen Konzepten am besten bin. Der Schwerpunkt meiner Karriere war, theoretische Konzepte über Mechanismen pathologischer Angst, Behandlungsmechanismen und Behandlungsresultate zusammenzubringen. Und ich wollte wissen, wann Patienten auf eine Behandlung nicht ansprechen, wann Patienten sich nicht erholen. Darin war ich gut, und ich fand, ich müsste nicht in jedem Bereich stark sein.
Was sind Ihrer Meinung nach die Hauptherausforderungen der Zukunft? Es ist natürlich immer schwierig, die Zukunft vorauszusagen, aber vielleicht die nahe Zukunft?

Edna Foa: Es gibt zwei Hauptherausforderungen. Bei Angstzuständen kennen wir viele Wege, Menschen zu helfen. Wir haben gute Therapien, auch wenn sie nicht vollkommen sind, nicht jeder davon profitiert und wir auch Misserfolge haben. Aber wir haben auch Misserfolge bei der Behandlung von Bluthochdruck oder Diabetes. Ich denke, bei Angststörungen sind wir wirklich gut. Misserfolge haben wir jedoch bei der Verbreitung unseres Wissens, zumindest in den Vereinigten Staaten. Wenn Sie Psychotherapeuten fragen: «Wie oft arbeiten Sie mit kognitiver Verhaltenstherapie?», werden Sie feststellen, dass nur sehr wenige damit arbeiten. Ich habe sogar den Verdacht, dass diejenigen, die damit arbeiten, es häufig falsch machen. Weil wir nicht nur insofern schlecht sind, dass wenige angeben, kognitiv verhaltenstherapeutisch zu arbeiten, sondern auch insofern, dass bei denjenigen, die so arbeiten, Therapietreue und -effektivität verloren gehen. Darum stecke ich so viel Arbeit in Workshops und Supervisionen, insbesondere mit Therapeuten, die mit Konfrontationstherapie arbeiten. Wir haben eine Methode entwickelt, um nicht nur das Behandlungsverfahren zu vermitteln, sondern auch langfristige Supervision bei der Anwendung. Aber wie gebe ich eine Behandlungsmethode an Hunderttausend Therapeuten weiter, ohne dass sie an Genauigkeit und Wirksamkeit verliert? Wir müssen also auch Behandlungen entwickeln, die leicht vermittelt werden können und gut funktionieren, selbst wenn sie nicht perfekt umsetzt werden. Konfrontationstherapie ist ziemlich anpassbar. Es ist einfach, das Prinzip zu verstehen. Kognitive Therapie ist komplizierter zu vermitteln, da ist es nicht so einfach, ihre ursprüngliche Wirksamkeit zu gewährleisten. Die andere Herausforderung ist, dass wir unser Wissen über kognitive Verzerrungen, über das Gedächtnis, über Wahrnehmung und über Kognitionen dazu benutzen, neue Behandlungen abzuleiten, die hoffentlich wirkungsvoller sein werden.

Eine letzte kurze Frage. Hat es Ihnen Spaß gemacht, dies alles zu tun?

Edna Foa: Diese ganze Arbeit? Ja, ich habe es wirklich genossen. Sobald Sie beschließen, Ihr Leben Ihrer Karriere zu widmen, beschließen Sie auch, in anderen Bereichen Ihres Lebens nicht Karriere zu machen. Vielleicht wäre ich auch gerne Architektin, ich liebe Design oder Innenarchitektur. Oder Archäologin. Es gibt eine Menge interessante Sachen, die ich getan haben könnte. Aber das ist immer so, nicht wahr? Die eine Möglichkeit schließt andere Möglichkeiten aus. Es ist wirklich sehr spannend, Experimente zu entwickeln, und es ist sehr, sehr aufregend, Patienten zu sehen, die sich innerhalb sehr kurzer Zeit erholen.

\section{Vielen Dank für das Gespräch!}

